\title{
Elongation Factor P: Function and Effects on Bacterial Fitness
}

Lili K. Doerfel, Marina V. Rodnina

Max Planck Institute for Biophysical Chemistry, Am Fassberg 1137077 Goettingen, Germany

Received 6 June 2013; accepted 19 June 2013

Published online 5 July 2013 in Wiley Online Library (wileyonlinelibrary.com). DOI 10.1002/bip.22341

\section{ABSTRACT:}

The elongation phase of translation is promoted by three universal elongation factors, EF-Tu, EF-Ts, and EF-G in bacteria and their homologs in archaea and eukaryotes. Recent findings demonstrate that the translation of a subset of $m R N A$ s requires a fourth elongation factor, EF-P in bacteria or the homologs factors a/eIF5A in other kingdoms of life. EF-P prevents the ribosome from stalling during the synthesis of proteins containing consecutive Pro residues, such as PPG, PPP, or longer Pro clusters. The efficient and coordinated synthesis of such proteins is required for bacterial growth, motility, virulence, and stress response. EF-P carries a unique post-translational modification, which contributes to its catalytic proficiency. The modification enzymes, which are lacking in higher eukaryotes, provide attractive new targets for the development of new, highly specific antimicrobials. (C) 2013 Wiley Periodicals, Inc. Biopolymers 99: 837-845, 2013.

Keywords: translation; ribosome; translation factors; $t R N A$

This article was originally published online as an accepted preprint. The "Published Online" date corresponds to the preprint version. You can request a copy of the preprint by emailing the Biopolymers editorial office at biopolymers@ wiley.com

Correspondence to: Marina V. Rodnina; e-mail: rodnina@mpibpc.mpg.de Contract grant sponsor: Max Planck Society, Deutsche Forschungsgemeinschaft (c) 2013 Wiley Periodicals, Inc.

\section{INTRODUCTION}

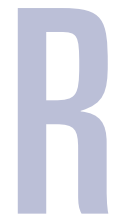

ibosomes are cellular machines that produce proteins in a rapid and accurate fashion. During the elongation phase of protein synthesis, the ribosome moves on the mRNA track, one codon at a time, with an average speed of about 10-20 codons per second (in bacteria). Each cycle of elongation entails three consecutive steps: (i) the binding of aminoacyl-tRNA (aatRNA) to its cognate codon exposed in the A site of the ribosome, (ii) peptide bond formation between aa-tRNA in the A site and peptidyl-tRNA in the P site, and (iii) translocation of the two tRNAs from the $\mathrm{A}$ to $\mathrm{P}$ and from the $\mathrm{P}$ to $\mathrm{E}$ site. The decoding and translocation reactions are aided by translation elongation factors (EF): EF-Tu accelerates aatRNA binding to the ribosome; EF-Ts is the guanine nucleotide exchange factor of EF-Tu, and EF-G promotes tRNA translocation. EF-Tu ensures that different aa-tRNAs are delivered to the ribosome at uniform rates, ${ }^{1}$ which should ensure that translation can proceed in a processive, rapid fashion independent of the mRNA codon. However, despite of the equalizing effect of EF-Tu, translation is kinetically discontinuous, with periods of rapid synthesis separated by pauses. $^{2}$ Reasons for translational pauses are diverse and not well understood. Pausing can be caused by e.g. rare codons, internal Shine-Dalgarno-like sequences in the mRNA, or mRNA secondary structures, among others. In a number of cases, ribosomes are stalled by specific leader peptides that interact with the ribosome exit tunnel and thereby control translation, e.g., secM, tnaC, mifM, erm $C L$, catA86L, arg2/CPA1, or human cytomegalovirus $g p 48{ }^{3}$ Additionally, ribosome pausing can be mediated by short sequences in nascent peptides that end with a Pro residue. ${ }^{4-}$ ${ }^{6}$ Pro and Gly are poor substrates for the peptidyl transfer reaction; both amino acids are incorporated into nascent proteins more slowly than any other amino acid and might thus lead to translational pauses. ${ }^{6-9}$ Recent findings demonstrate that ribosome stalling on consecutive Pro codons is alleviated by a noncanonical elongation factor, EF-P. ${ }^{10,11}$ EF-P so far is the only translation factor that can augment the peptidyl transferase activity of the ribosome. In this 


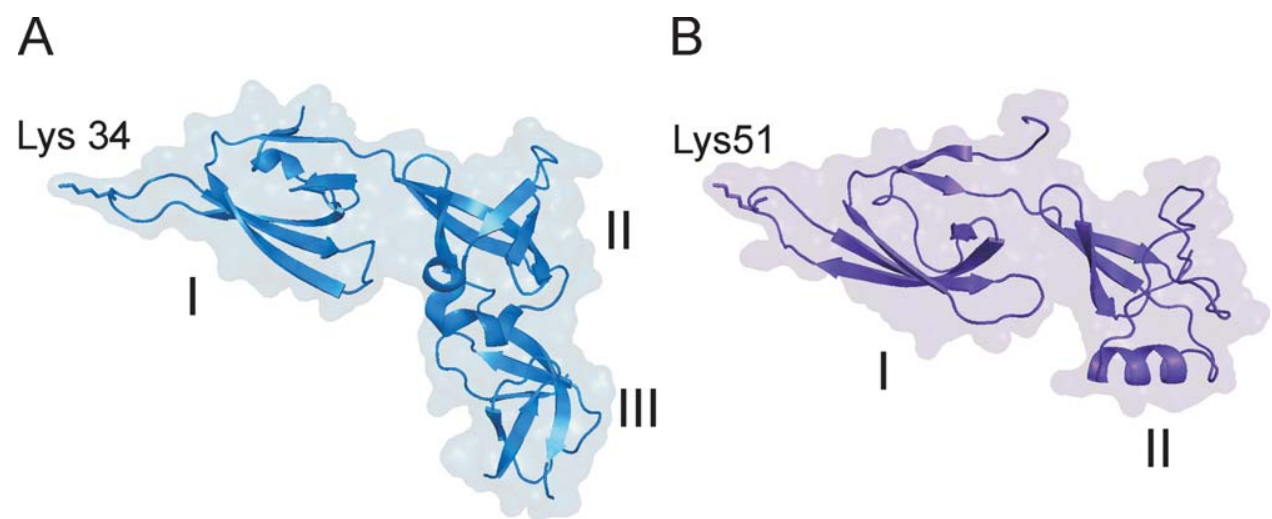

FIGURE 1 Structures of EF-P and eIF5A. (A) EF-P from E. coli (PDB 3A5Z) ${ }^{14}$. Domains I, II, and III and the functionally important Lys34 residue are indicated. (B) eIF5A from S. cerevisiae (PDB 3ER0) with domains I and II and the conserved Lys51 residue.

review, we describe the structure and function of EF-P and discuss its importance for the fitness of bacteria.

\section{STRUCTURE AND POST-TRANSLATIONAL MODIFICATION}

\section{Structure}

EF-P is a $21 \mathrm{kDa}$ protein encoded by the efp gene, which maps to the $94.3 \mathrm{~min}$ on the Escherichia coli chromosome. ${ }^{12,13}$ EF-P and its archaeal/eukaryotic orthologs a/eIF5A are universally conserved proteins. EF-P is composed of three $\beta$-barrel domains and mimics a tRNA in shape and size ${ }^{14-16}$ (Figure 1A). The C-terminal domain III of bacterial EF-P possesses the same fold as domain II and might be the result of a partial gene duplication event. ${ }^{14}$ eIF5A also has a tRNA-like shape ${ }^{17}$ (Figure 1B). aIF5A and eIF5A share $84 \%$ and $64 \%$ sequence similarity, respectively, ${ }^{18}$ with the part of EF-P comprising domains I and II. It is not clear why eIF5A does not have domain III. It has been proposed that eIF5A dimerizes producing a molecular envelope similar to the EF-P monomer. ${ }^{17}$ The crystal structure of Thermus thermophilus EF-P bound to
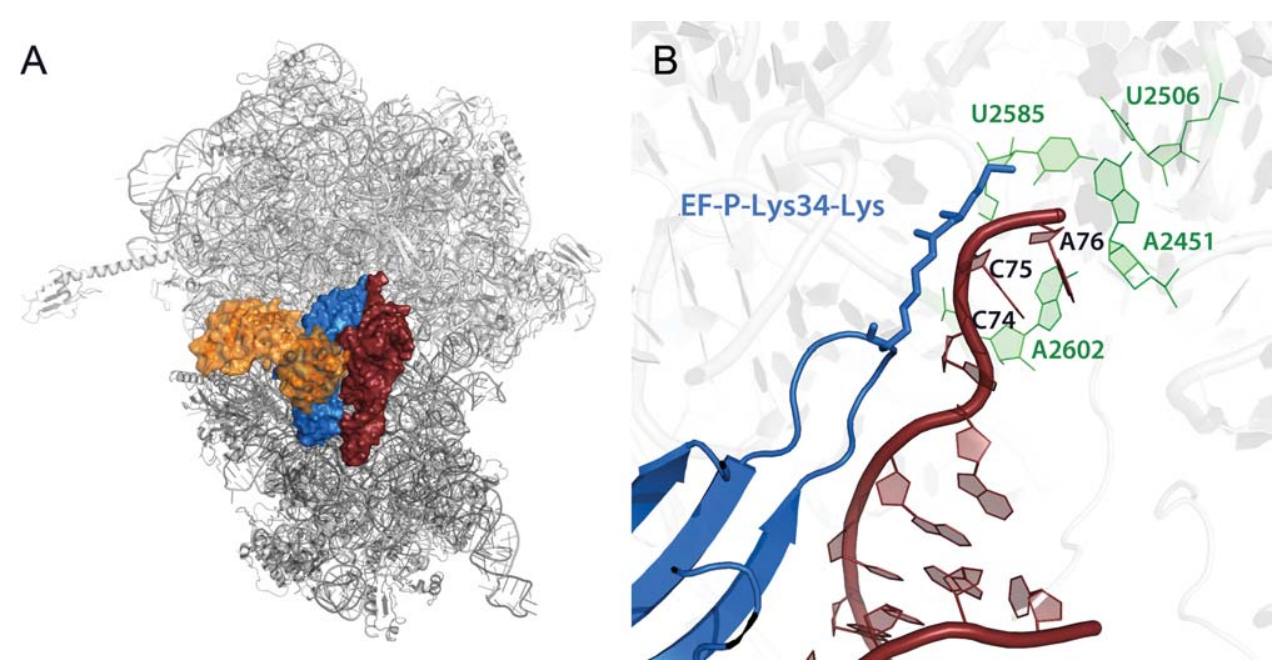

FIGURE 2 Crystal structure of the ribosome-EF-P complex. ${ }^{19}$ (A) EF-P from T. thermophilus (PDB 3HUW and 3HUX) binds between the $\mathrm{E}$ and the $\mathrm{P}$ site of the ribosome and contacts tRNA ${ }^{\mathrm{fMet}}$. The $50 \mathrm{~S}$ and $30 \mathrm{~S}$ subunits are colored in light and dark gray, respectively. EF-P is shown in blue and $\mathrm{tRNA}^{\mathrm{fMet}}$ in dark red. Ribosomal protein L1 is shown in orange. (B) A model of lysinylated EF-P bound to the ribosome. Color code as in (A). 23S rRNA residues at the catalytic core of the ribosome (A2451, U2506, U2585, and A2602) are depicted in green. 
A

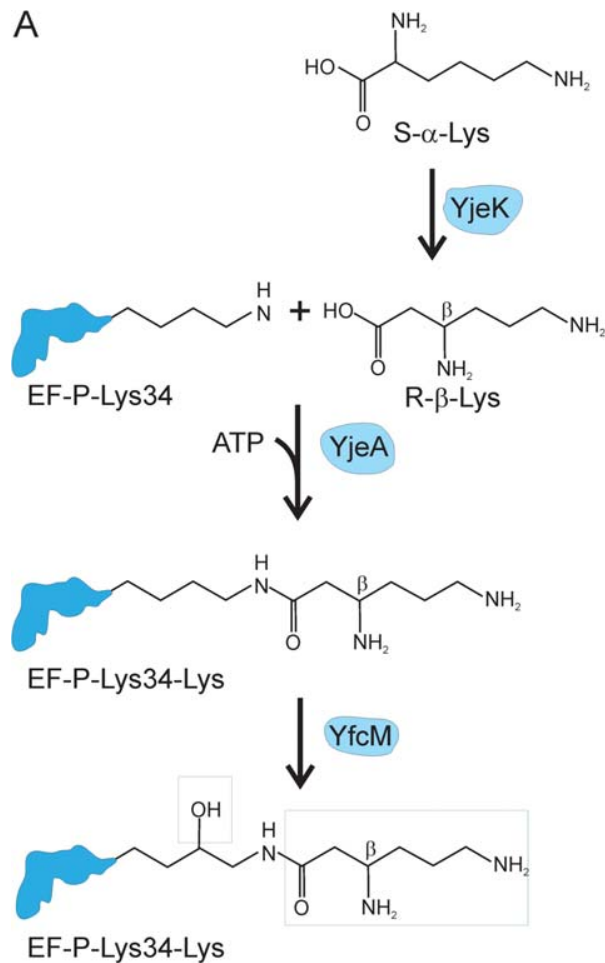

B
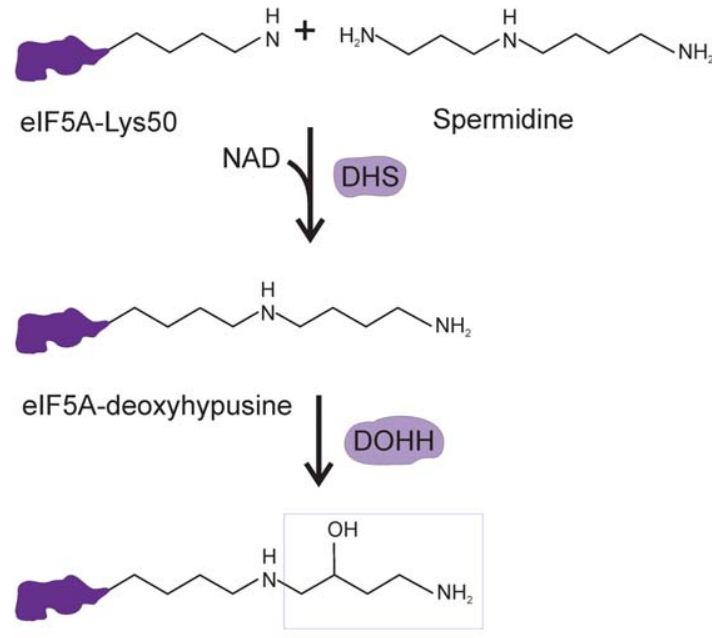

elF5A-hypusine

FIGURE 3 Modification pathways of EF-P and eIF5A. (A) In bacteria, YjeK converts free S- $\alpha$-Lys to R- $\beta$-Lys, which is then transferred by YjeA to the $\varepsilon$-amino group of Lys34 of EF-P. Subsequently, YfcM hydroxylates Lys34, presumably at its C5 $(\delta)$. (B) In S. cerevisiae (and other eukaryotes), deoxyhypusine synthase (DHS) transfers the aminobutyl moiety of spermidine to Lys51 of eIF5A. Deoxyhypusine hydroxylase (DOHH) hydroxylates the deoxyhypusine intermediate to form hypusine [N $\mathrm{N}^{\varepsilon}$-(4-aminobutyl-2-hydroxy)-1-Lys].

the ribosome with the initiator tRNA ${ }^{\mathrm{fMet}}$ in the $\mathrm{P}$ site shows EF-P bound at the interface of the $30 \mathrm{~S}$ and $50 \mathrm{~S}$ subunits between the P site and E site. Domains III, II, and I of EF-P interact with tRNA ${ }^{\text {fMet }}$ near its anticodon stem-loop, the D loop, and the acceptor end in close proximity of the peptidyl transferase center, respectively (Figure 2A). ${ }^{19}$ The network of interactions between EF-P and tRNA ${ }^{\mathrm{fMet}}$ appears to stabilize the tRNA, which may be one of the factor's functions on the ribosome. ${ }^{10,19}$ In the ribosome-EF-P complex, the L1 stalk has moved into the E site and contacts domain II of EF-P. ${ }^{19}$ The L1 stalk is a dynamic component of the 50S subunit, which is involved in tRNA translocation and release. ${ }^{20-22}$ The role of the EF-P-L1 interaction for EF-P recruitment or function is not clear. While T. thermophilus EF-P has an Arg at the tip of its N-terminal domain, ${ }^{19}$ E. coli EF-P harbors a conserved Lys residue (position 34), which is post-translationally modified by lysinylation and hydroxylation ${ }^{18,23-26}$ and may protrude even further into the peptidyl transferase center (Figure 2B).

\section{Post-Translational Modification}

The post-translational modification of EF-P requires the action of three enzymes: YjeA (also known as PoxA or GenX), YjeK, and $\mathrm{YfcM}^{23-27}$ (Figure 3A), resulting in lysinylation and hydroxylation of Lys34 in E. coli EF-P. Epistatic analysis of the expression of yjeA and yjeK genes in Salmonella typhimurium suggested their sequential involvement in the modification pathway. ${ }^{27,28}$ However, about $70 \%$ of bacteria do not encode YjeA or YjeK, although most of them have a Lys residue at the position homologs to Lys34 in E. coli, suggesting that in these bacteria EF-P is either not modified or modified in a different way. ${ }^{26}$ Some organisms have two EF-P paralogs, one with the conserved Lys and the other in which the Lys residue has been replaced by e.g. Ala or His. ${ }^{26}$ In archaeal and eukaryotic IF5A there is a Lys at homologs position, which is also modified, although the modification is quite different, to hypusine $\left[\mathrm{N}^{\varepsilon}-\right.$ (4-aminobutyl-2-hydroxy)-l-lysine] by an unrelated pathway (Figure 3B). Hypusination of a/eIF5A is ubiquitous among archaea and eukarya and is required for eIF5A function and cell viability in yeast. ${ }^{29}$ 
A

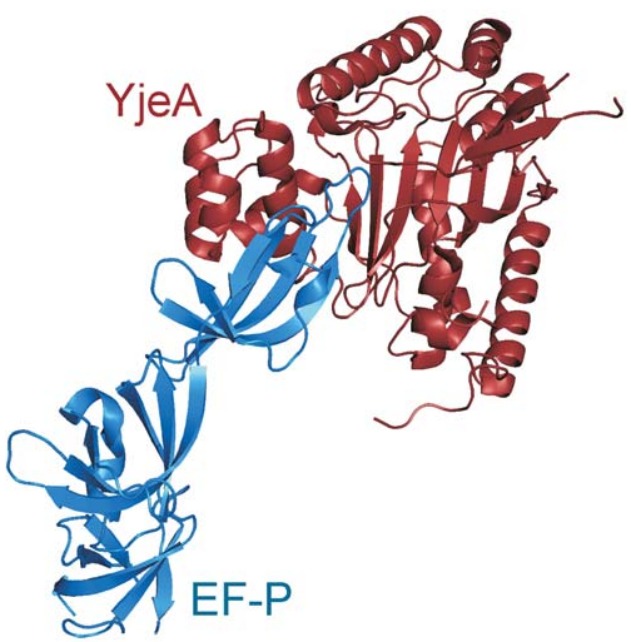

B

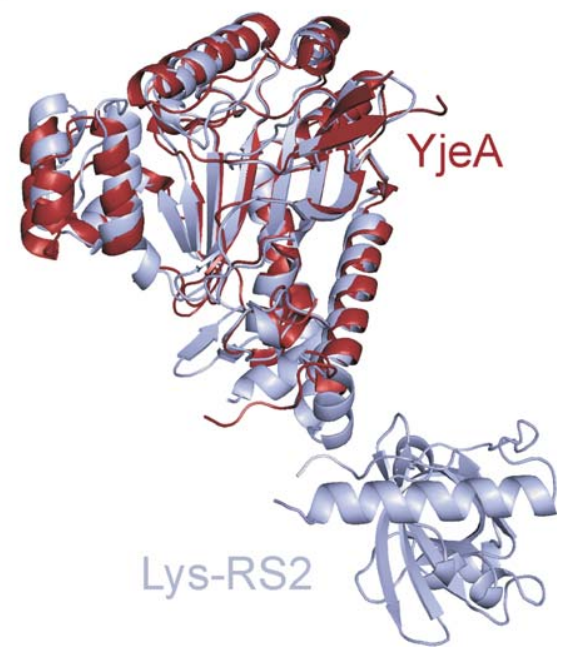

FIGURE 4 YjeA as ortholog of Lys-tRNA synthetases. (A) E. coli EF-P (shown in blue) bound to YjeA (red) (PDB ID 3A5Z). ${ }^{31}$ (B) Superimposition of E. coli YjeA (PDB 3A5Z shown in red) ${ }^{24}$; and E. coli Lys-tRNA synthetase (PDB 1BBU, shown in gray). ${ }^{31}$

YjeK is a homolog of lysine 2, 3 aminomutase (LAM), but it lacks the multimerization domain found in that protein family. While LAM proteins usually promote the conversion of S$\alpha$-Lys to S- $\beta$-Lys, $E$. coli YjeK converts S- $\alpha$-Lys to R- $\beta$-Lys, ${ }^{26,30}$ which is the first step on the EF-P modification pathway. YjeA is a paralog of the lysyl-tRNA synthetases (Lys-RS2), which charge tRNA ${ }^{\text {Lys }}$ with Lys. Sequence and structure of the catalytic cores of YjeA and Lys-RS2 are very similar ${ }^{24-26}$ (Figure 4A); however, YjeA does not have the anticodon binding domain, which is important for tRNA ${ }^{\text {Lys }}$ recognition by LysRS2, and does not aminoacylate any tRNA with Lys. ${ }^{24,25}$ Instead, YjeA utilizes R- $\beta$-Lys produced by YjeK, forms an activated Lys-adenylate, and transfers the Lys moiety to the $\varepsilon^{-}$ amino group of Lys 34 of EF-P (Figure 4B). ${ }^{24}$ YjeA from $S$. thyphimurium activates S- $\beta$-Lys 100 -fold more efficiently than $\alpha$ Lys. $^{32}$ Furthermore, supplementing the medium with $\beta$-Lys, but not $\alpha$-Lys, can substitute for YjeK function in vivo. ${ }^{32}$ These findings indicate that the synthesis of $\beta$-Lys by YjeK occurs before its addition to EF-P by YjeA. Lysinylated EF-P is additionally modified by $\mathrm{YfcM}$, which hydroxylates the conserved Lys34, presumably at its $\mathrm{C} 5(\delta) .{ }^{23}$ It was proposed to rename YjeA, YjeK, and Yfcm to EF-P-modifying enzymes (Epm) EpmA, EpmB and EpmC, respectively. ${ }^{23}$

In eukaryotes eIF5A is modified by deoxyhypusine synthase (DHS) and deoxyhypusine hydroxylase (DOHH). DHS transfers the aminobutyl moiety of spermidine to Lys51 of eIF5A from Saccharomyces cerevisiae in an NAD-dependent manner, thereby generating a deoxyhypusine [ $N^{\varepsilon}$-(4-aminobutyl)-1lysine] intermediate. Subsequently, DOHH completes hypusine
[ $\mathrm{N}^{\varepsilon}$-(4-aminobutyl-2-hydroxy)-1-lysine] synthesis by hydroxylation of the deoxyhypusine intermediate. ${ }^{33}$ While only DHS is essential in yeast, DOHH is essential in higher eukaryotes, e.g., in Caenorhabditis elegans or Drosophila melanogaster. Two or more eIF5A isoforms have been identified in eukaryotes. ${ }^{34}$ In archaea, DHS, but not DOHH, could be identified so far, ${ }^{35}$ although both deoxyhypusinated and hypusinated forms of aIF5A were found ${ }^{34}$; the putative hydroxylase that acts on aIF5A is unknown. DHS and DOHH homologs are absent in most bacteria, ${ }^{36}$ and only a few contain a DHS-related gene, probably due to lateral gene transfer from an archaeon. ${ }^{33}$

\section{FUNCTION}

\section{The Long Way Towards Understanding EF-P}

\section{Function}

EF-P was discovered in 1975 by Glick and Ganoza ${ }^{37}$ as a factor that increased the yield of peptide bond formation between initiator fMet-tRNA ${ }^{\mathrm{fMet}}$ and a mimic of aa-tRNA, puromycin (Pmn). The effect of the factor was abolished by antibiotics known to specifically block peptide bond formation, suggesting that EF-P acts at the peptidyl transferase center of the ribosome. ${ }^{38,39}$ This prompted the authors to name the newly discovered protein EF-P, a factor that stimulates the peptidyl transferase activity of the ribosome. ${ }^{37}$ On the other hand, EF-P did not stimulate poly(Phe) or poly(Lys) synthesis ${ }^{40,41}$ and turned out to be dispensable for the synthesis of model proteins in reconstituted in vitro translation systems. ${ }^{42}$ 


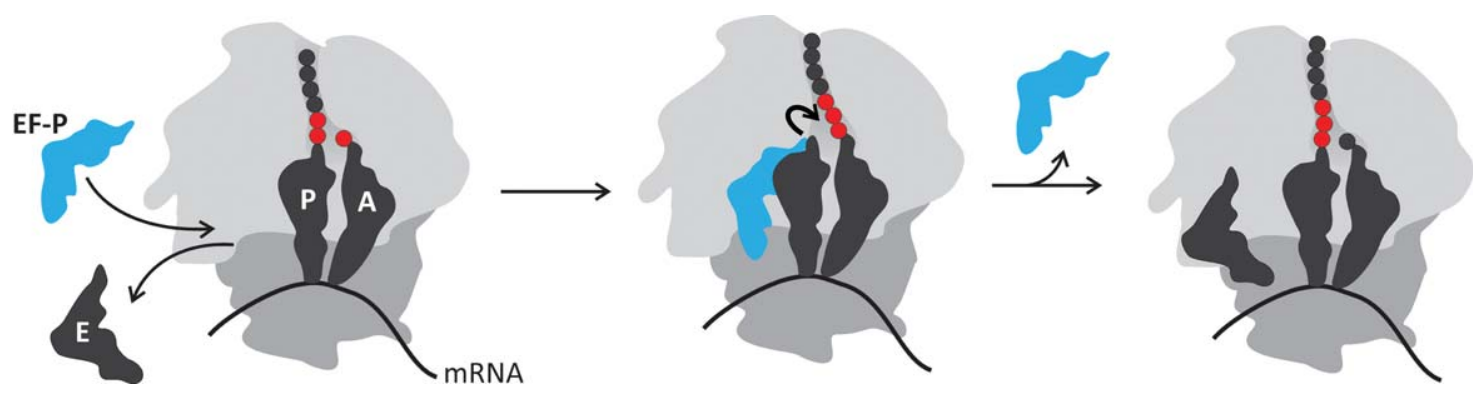

FIGURE 5 Mechanism of EF-P action on the ribosome. Left panel, the ribosome stalls after incorporation of two consecutive Pro residues with Pro or Gly as the next incoming amino acid; the E site becomes free after dissociation of deacylated tRNA, which allows for binding of EF-P. Middle panel, EF-P accommodates between $\mathrm{E}$ and $\mathrm{P}$ sites and promotes rapid peptide bond formation. Right panel, following the dissociation of EF-P translation can resume.

Furthermore, EF-P is present at about 0.1 copies per ribosome in E. coli, ${ }^{13}$ although it forms a 1:1 complex with ribosomes, ${ }^{18}$ making its involvement in each round of peptide bond formation unlikely. These results led to the suggestion that EF-P promotes the synthesis of the first peptide bond only. ${ }^{37}$ Likewise, eIF5A was identified as a factor acting during formation of the first peptide bond. ${ }^{43,44}$ This view was challenged by Saini et al., ${ }^{45}$ who have shown that yeast eIF5A promotes elongation rather than initiation, suggesting that EF-P might have a function during elongation as well. In fact, EF-P was shown to act on elongator aa-tRNAs analogs with a preference for those that were poor peptide bond acceptors. ${ }^{41,46}$ Furthermore, the accumulation of polysomes in E. coli harboring mutant EF-P, YjeA, or YjeK is consistent with a function of EF-P in elongation. ${ }^{40}$ This evidence prompted several groups to re-examine the function of EF-P, which led to a new insight into the cellular role of EF-P and a/eIF5A.

\section{EF-P Alleviates Ribosome Pausing at Pro}

Our motivation to study the effect of EF-P on translation arose through the efforts to understand how Pro can be rapidly incorporated into proteins in the cell, given that in vitro Pro renders peptide bond formation extremely slow. ${ }^{6-9}$ For example, the rate of the Pmn reaction with Pro is $0.01 s^{-1} ; 10-1000$ times slower than with other amino acids. We found that EF-P accelerates the reaction between $\mathrm{fMetPro}_{\mathrm{tRNA}}{ }^{\text {Pro }}$ and Pmn by 90-fold. ${ }^{10}$ Furthermore, EF-P enhances the incorporation of Gly and Pro into peptides by 8 -fold and 15-fold, respectively, and stabilizes the tRNA binding in the $\mathrm{P}$ site, thereby preventing its loss from stalled ribosomes. ${ }^{10}$ In parallel, Ude et al. ${ }^{11}$ came to similar conclusions while searching for new regulatory players of the Cad module, a lysine-dependent acid-resistance system in bacteria. They found that translation of CadC, a transcriptional regulator containing a Pro cluster, was impaired in strains where the genes for EF-P or its modification enzymes were disrupted. Both groups showed that peptide bond formation with several consecutive Pro residues causes the ribosome to stall and that EF-P rescues the synthesis by alleviating stalling and facilitating rapid incorporation of Pro residues into peptides ${ }^{10,11}$ (Figure 5). Three Pro residues in a row are sufficient to induce ribosome stalling at the second Pro codon ${ }^{5,11}$ while the incorporation of the third Pro is impaired. Engineering PPP or PPG motifs into a protein, which originally does not contain such sequences and is rapidly synthesized independent of the presence of EF-P, causes stalling that is rescued by EF-P. ${ }^{10}$ The amino acid itself, rather than the codon, is responsible for the dependence on EF-P, ${ }^{11}$ and all Pro-coding triplets can cause stalling. In E. coli, about 270 proteins have PPG and PPP motifs or contain longer Pro sequences, and their synthesis may depend on EF-P action. In fact, such motifs are overrepresented in proteins that are down-regulated in an EF-P deletion strain (efp ${ }^{-}$) of S. typhimurium. ${ }^{47}$ Thus, EF-P is an elongation factor, which is required for the rapid synthesis of a subset of cellular proteins by augmenting the peptidyl transferase activity of the ribosome. ${ }^{10,11} \mathrm{~A}$ recent article shows that eIF5A from yeast has the same function. ${ }^{48}$

Although stalling of ribosomes on poly(Pro) stretches and the rescue effect of EF-P was convincingly demonstrated for a number of proteins, apparently not all poly(Pro)-containing proteins are inefficiently synthesized in the efp strain of $S$. typhimurium. ${ }^{47}$ Because the proteome analysis reports on steady-state levels of proteins, some of the EF-P effects might have been missed, e.g., in those cases where the translation can proceed without EF-P, albeit at reduced rates, such as demonstrated for YafD and TonB. ${ }^{10}$ A basal level of product formation might explain why efp ${ }^{-}$strains are viable. The kinetic effect of EF-P may be more pronounced at conditions which require rapid remodeling of the cellular proteome to the changing environment, e.g. stress conditions. Proteomic analysis also 
revealed that the deletion of EF-P influences proteins which contain motifs other than PPP and PPG, such as APP, YIRYIR, and GSCGPG, or even no discernible motifs at all. ${ }^{47} \mathrm{~A}$ more comprehensive search would be desirable to identify other amino acid combinations that are sensitive to EF-P.

The reason for the impaired peptidyl transferase reaction with Pro residues is unclear. Among the proteinogenic amino acids proline is the only secondary amine. Its ring structure, which restricts its rotation angles, may impose structural constraints that hinder peptide bond formation. EF-P might help the ribosome and the peptidyl- and aa-tRNA substrates in the peptidyl transferase center to adopt a conformation/position which is more accessible for nucleophilic attack. ${ }^{10}$ Another role for EF-P, particularly when the nascent peptide is still short, may be to prevent the drop-off of peptidyl-tRNA from stalled ribosomes, which would increase the yield of peptide. ${ }^{10}$ Notably, the pausing itself may be a prerequisite for EF-P binding. While in the processively working ribosomes, the tRNAs permanently transit through the $\mathrm{A}, \mathrm{P}$, and $\mathrm{E}$ sites, thereby making the $\mathrm{E}$ site inaccessible for EF-P binding, the release of deacylated tRNA from the $\mathrm{E}$ site during the elongation round preceding stalling may provide a window of opportunity for EF-P to bind.

In vivo, lysinylation is crucial for EF-P function in those organisms in which the modification is possible, ${ }^{27,28,40,49}$ while the hydroxylation seems dispensable. ${ }^{40}$ An EF-P(K34A) mutant, in which Lys34 was replaced with Ala and hence cannot be modified, does not rescue the efp phenotype in $E$. coli. $^{24}$ On the other hand, the deletion of EF-P has more severe effects in vivo than the deletion of the modifying enzymes responsible for lysinylation (YjeA and YjeK). ${ }^{27,28,40,49}$ This suggests that lysinylation is important, but not essential for EF-P function. In vitro, unmodified EF-P and EF-P(K34A) show reduced activity, ${ }^{10,11,40,50}$ which can be fully restored by lysinylation. ${ }^{10}$ The modification increases both the affinity of EF-P binding to the ribosome (30-fold) and the maximal rate of peptide bond formation (4-fold), leading to an 120-fold increase in $k_{\text {cat }} / K_{\mathrm{M}} \cdot{ }^{10}$ Thus, the modifications substantially improve the catalytic proficiency of EF-P. A basal activity level might nevertheless be sufficient to support translation in those organisms which do not encode modifying enzymes. Because the modification increases the affinity of EF-P binding to the ribosome, one could speculate that the effect of EF-P could also be achieved if the concentration of the unmodified factor in the cell were higher than in E. coli.

\section{IMPORTANCE FOR BACTERIAL FITNESS}

Although EF-P is not an essential protein, deletions of EF-P or its modifying enzymes YjeA or YjeK lead to pleiotropic pheno- types, which are similar in different organisms, such as E. coli, ${ }^{28,51-53}$ S. typhimurium, ${ }^{27,28,54,55}$ Agrobacterium tumefaciens, ${ }^{56,57}$ Bacillus subtilis, ${ }^{58}$ Brucella abortus, ${ }^{59}$ or Pseudomonas aeruginosa, ${ }^{53}$ and these phenotypes can be rescued by EF-P expressed in trans. ${ }^{51,57,59}$ The mutations lead to reduced growth rate and motility, ${ }^{49,51-54,56,57,59}$ susceptibility to hyperosmotic conditions and different stress factors, such as acids, detergents and antibiotics, ${ }^{27,49,53,55,60}$ enhanced metabolism, ${ }^{27,55}$ and reduced virulence. ${ }^{54-57,59}$ While there seem to be no influence on transcription, the translation appears to be affected, ${ }^{51,55}$ resulting in an altered expression of a number of proteins. $^{27,53,55}$ Frequently, metabolic proteins, membraneassociated proteins, transporters and two-component regulatory systems, especially those involved in chemotaxis and motility, display changed expression levels in the deletion strains, whereas proteins belonging to the basal transcriptiontranslation machinery are rarely affected. ${ }^{27,47}$

Many of the observed pleiotropic effects of mutations in the efp, yjeA, or $y j e K$ genes can be rationalized by the aberrant synthesis of proteins with proline stretches, leading to changes in metabolic networks. EF-P may be critical for the copy-number adjustment of multiple pathways, thereby contributing to the fitness of the bacterial proteome. For example, by facilitating the synthesis of CadC, EF-P affects the function of the twocomponent regulatory Cad module. ${ }^{11} \mathrm{~A}$ decreased synthesis of TonB,${ }^{10}$ which supplies the energy for TonB-dependent transporters, may result in the cumulative inhibition of transport processes. ${ }^{61}$ The reduced motility of cells deficient in EF-P modification enzymes can be explained by an inefficient translation of the flagellar proteins FlhC, FlK, or FliF, which all contain PPP and PPG motifs. In fact, the synthesis of FlhC and Flk is reduced in the efp ${ }^{-}$strain of S. typhimurium due to stalling at a poly(Pro) stretch. ${ }^{11}$ Synthesis of CheA, another protein involved in motility and chemotaxis, is down-regulated in the $\mathrm{efp}^{-}$strain of $S$. thyphimurium. It contains an APP motif, which can cause stalling. ${ }^{47} E f p^{-}, y j e A^{-}$, and $y j e K^{-}$strains of $S$. typhimurium are unable to use $\gamma$-glutamyl-glycine as nitrogen source and are resistant to S-nitroso-glutathion (GSNO), ${ }^{27,49}$ which is cytostatic for S. typhimurium. ${ }^{62}$ Proteomic analysis revealed that gamma-glutamyl transferase (Ggt), which contains a PPP motif, is decreased in the $e f p^{-}$strain. Because the enzyme is required for the utilization of $\gamma$-glutamyl-glycine, impaired synthesis of Ggt leads to GSNO resistance. ${ }^{47}$

Numerous examples indicate that mutations of the efp, $y j e A$, and $y j e K$ genes result in reduced virulence of a wide range of pathogenic bacteria, and there are some examples where EF$\mathrm{P}$ directly affects the synthesis of proteins linked to virulence. For example, translation of EspF, a key player during the infection by enterohemorrhagic and enteropathogenic E. coli strains (EHEC and EPEC) ${ }^{63,64}$ is stalled at a PPPP stretch in the 


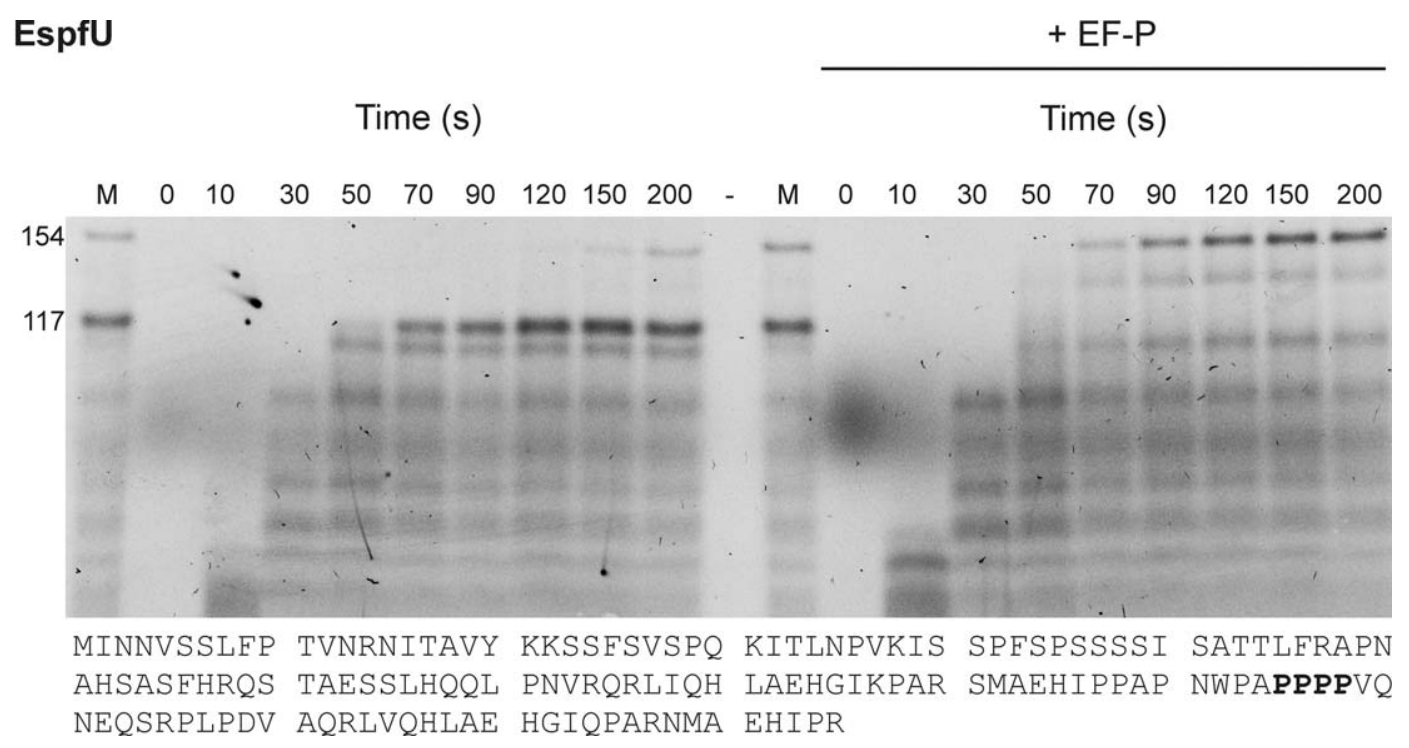

FIGURE 6 Alleviation by EF-P of ribosome stalling on the PPPP sequence in EspfU. Translation of an N-terminal fragment of EspfU (154 amino acids) and peptide separation on SDS-page was carried out as described. ${ }^{10}$ Peptides were visualized by the fluorescence of BODIPY-FL attached to their $\mathrm{N}$-terminus. $\mathrm{M}$ denotes EspfU fragments of the indicated amino acids lengths.

absence of EF-P, and the translational arrest is alleviated by EF$\mathrm{P}$ (Figure 6). Given the low catalytic proficiency of unmodified EF-P and the fact that modification pathways use different enzymes in prokaryotes and eukaryotes, the bacterial modifying enzymes might be interesting targets for new antibiotics. ${ }^{10}$

The effects of deletions of EF-P or EF-P-modification enzymes may also affect virulence indirectly by changing the overall balance in the proteome. In $p o x A^{-}\left(y j e A^{-}\right)$strains of $S$. typhimurium, a class of proteins associated with the SPI-1 pathogenicity island and the transcription factor HilA regulating SPI-1 are overexpressed. ${ }^{27}$ SPI-1 expression mediates invasion of host cells and is down-regulated in the wild-type S. typhimurium after the internalization by the host macrophage. Persistent expression of SPI-1 proteins in macrophages is cytotoxic ${ }^{65,66}$ and detrimental to bacterial growth within the host. ${ }^{67,68}$ Interestingly, the pox $A^{-}$strains are still immunogenic and can therefore be used to generate effective antibodies against their wild-type counterpart. ${ }^{54}$ Another protein overexpressed in the $p o x A^{-}$strain is the virulence factor $\mathrm{PhoP}{ }^{55}$ which belongs to the two-component system PhoP/PhoQ. Constitutively, expression of PhoP impairs virulence and survival of S. typhimurium in mammalian hosts. ${ }^{69}$ Examples of proteins whose expression is down-regulated in cells lacking modified EF-P are outer-membrane proteins such as KdgM and SlyB in the efp ${ }^{-}$strain of S. typhimurium, ${ }^{49} \mathrm{MexX}$, a component of RND family efflux pump, in $e f p^{-}$strain of $P$. aeroginosa, ${ }^{53} \operatorname{ManX}(\mathrm{YZ})$ in the pox $A^{-}$strain of S. typhimurium, ${ }^{27}$ a mannose-specific IIAB component required for S. typhimu- rium growth in macrophages, ${ }^{70}$ or the virulence factor VirE2 in A. tumefaciens. ${ }^{57}$

While pleiotropic effects of EF-P on different aspects of bacterial physiology become increasingly clear, the importance of eIF5A is less well understood. Unlike EF-P, aIF5A/eIF5A are essential proteins, ${ }^{71}$ which may correlate with a high abundance of proteins with Pro clusters. eIF5A was implicated in a broad range of cellular processes such as mRNA decay, nucleocytoplasmic transport, and cell cycle progression. ${ }^{34,72}$ Interestingly, both human eIF5A isoforms are associated with aberrant cell proliferation: isoform 1 is important for the active proliferation of various cancer cells, whereas isoform 2 is upregulated in e.g., ovarian and colorectal cancer cell lines. This underlines the importance of eIF5A for the proper cell function and provides yet another potential application for eIF5A in biomedical research.

\section{CONCLUSIONS}

The discovery of the EF-P function marks a change in the inventory of translation factors. The initiation phase of translation requires a different set of factors in bacteria (only three, IF1, IF2, and IF3; for recent review) ${ }^{73}$ and eukaryotes (at least nine). ${ }^{74}$ In contract to initiation, the elongation phase is highly conserved with five EF involved (EF-Tu, EF-Ts, EF-G, EF-P, and SelB in bacteria and their homologs in eukaryotes). Termination requires RF1/2 and RF3 in bacteria and only two factors, RF1 and RF3, in eukaryotes. Finally, the ribosome recycling phase requires only the ribosome recycling factor 
(RRF) and EF-G in bacteria, whereas in eukaryotes RRF is lacking and the reaction is facilitated by the action of $\mathrm{ABCE} 1$, eIF3, 3j, eIF1, eIF1A, and Ligatin. ${ }^{75}$ This clearly demonstrates the conservation of the elongation mechanism and provides insights into the evolution of the translational apparatus. In addition to EF-Tu, EF-Ts, and EF-G, which act in each round of translation elongation, EF-P and SelB are specialized factors that act on a subset of substrates. While SelB has emerged to deliver a single amino acid, selenocysteine, to the ribosome, EF-P is necessary to accelerate translation when several Pro residues are incorporated. Pro, (together with Ala, Glu, and Gly) tends to be lost during the evolution, ${ }^{76}$ possibly because it stalls the translation. The emergence of a factor that specifically accelerated Pro incorporation might result from an evolutionary trade-off between the crucial role of Pro clusters in some proteins and the requirement for rapid, processive protein synthesis. One can envisage that the specific requirements of eukaryotic cells, particularly with respect to translational control, compartmentalization of protein synthesis, and larger protein domains, might lead to emergence of additional, yet uncharacterized proteins that modify the functions of the ribosome or of the universal translation factors. If true, identification and understanding of this auxiliary machinery would be a very exciting avenue for future research.

\section{REFERENCES}

1. Ledoux, S.; Uhlenbeck, O. C. Mol Cell 2008, 31, 114-123.

2. Li, G. W.; Oh, E.; Weissman, J. S. Nature 2012, 484, 538-541.

3. Wilson, D. N.; Beckmann, R. Curr Opin Struct Biol 2011, 21, 274-282.

4. Tanner, D. R.; Cariello, D. A.; Woolstenhulme, C. J.; Broadbent, M. A.; Buskirk, A. R. J Biol Chem 2009, 284, 34809-34818.

5. Woolstenhulme, C. J.; Parajuli, S.; Healey, D. W.; Valverde, D. P.; Petersen, E. N.; Starosta, A. L.; Guydosh, N. R.; Johnson, W. E.; Wilson, D. N.; Buskirk, A. R. Proc Natl Acad Sci U S A 2013, 110, E878-E887.

6. Muto, H.; Ito, K. Biochem Biophys Res Commun 2008, 366, 1043-1047.

7. Wohlgemuth, I.; Brenner, S.; Beringer, M.; Rodnina, M. V. J Biol Chem 2008, 283, 32229-32235.

8. Pavlov, M. Y.; Watts, R. E.; Tan, Z.; Cornish, V. W.; Ehrenberg, M.; Forster, A. C. Proc Natl Acad Sci U S A 2009, 106, 50-54.

9. Johansson, M.; Ieong, K. W.; Trobro, S.; Strazewski, P.; Aqvist, J.; Pavlov, M. Y.; Ehrenberg, M. Proc Natl Acad Sci U S A 2011, 108, 79-84.

10. Doerfel, L. K.; Wohlgemuth, I.; Kothe, C.; Peske, F.; Urlaub, H.; Rodnina, M. V. Science 2013, 339, 85-88.

11. Ude, S.; Lassak, J.; Starosta, A. L.; Kraxenberger, T.; Wilson, D. N.; Jung, K. Science 2013, 339, 82-85.

12. Aoki, H.; Adams, S. L.; Chung, D. G.; Yaguchi, M.; Chuang, S. E.; Ganoza, M. C. Nucleic Acids Res 1991, 19, 6215-6220.

13. An, G.; Glick, B. R.; Friesen, J. D.; Ganoza, M. C. Can J Biochem 1980, 58, 1312-1314.
14. Hanawa-Suetsugu, K.; Sekine, S.; Sakai, H.; Hori-Takemoto, C.; Terada, T.; Unzai, S.; Tame, J. R.; Kuramitsu, S.; Shirouzu, M.; Yokoyama, S. Proc Natl Acad Sci U S A 2004, 101, 9595-9600.

15. Choi, S.; Choe, J. Proteins 2011, 79, 1688-1693.

16. Kristensen, O.; Laurberg, M. Acta Crystallogr D Biol Crystallogr 2002, 58, 1039-1041.

17. Dias, C. A.; Garcia, W.; Zanelli, C. F.; Valentini, S. R. Amino Acids 2013, 44, 631-644.

18. Aoki, H.; Xu, J.; Emili, A.; Chosay, J. G.; Golshani, A.; Ganoza, M. C. FEBS J 2008, 275, 671-681.

19. Blaha, G.; Stanley, R. E.; Steitz, T. A. Science 2009, 325, 966-970.

20. Fei, J.; Kosuri, P.; MacDougall, D. D.; Gonzalez, R. L., Jr. Mol Cell 2008, 30, 348-359.

21. Munro, J. B.; Altman, R. B.; Tung, C. S.; Cate, J. H.; Sanbonmatsu, K. Y.; Blanchard, S. C. Proc Natl Acad Sci U S A 2010, 107, 709-714.

22. Valle, M.; Zavialov, A.; Sengupta, J.; Rawat, U.; Ehrenberg, M.; Frank, J. Cell 2003, 114, 123-134.

23. Peil, L.; Starosta, A. L.; Virumae, K.; Atkinson, G. C.; Tenson, T.; Remme, J.; Wilson, D. N. Nat Chem Biol 2012, 8, 695-697.

24. Yanagisawa, T.; Sumida, T.; Ishii, R.; Takemoto, C.; Yokoyama, S. Nat Struct Mol Biol 2010, 17, 1136-1143.

25. Ambrogelly, A.; O’Donoghue, P.; Soll, D.; Moses, S. FEBS Lett 2010, 584, 3055-3060.

26. Bailly, M.; de Crecy-Lagard, V. Biol Direct 2010, 5, 3.

27. Navarre, W. W.; Zou, S. B.; Roy, H.; Xie, J. L.; Savchenko, A.; Singer, A.; Edvokimova, E.; Prost, L. R.; Kumar, R.; Ibba, M.; Fang, F. C. Mol Cell 2010, 39, 209-221.

28. Zou, S. B.; Roy, H.; Ibba, M.; Navarre, W. W. Virulence 2011, 2, 147-151.

29. Park, M. H.; Joe, Y. A.; Kang, K. R. J Biol Chem 1998, 273, 1677-1683.

30. Behshad, E.; Ruzicka, F. J.; Mansoorabadi, S. O.; Chen, D.; Reed, G. H.; Frey, P. A. Biochemistry 2006, 45, 12639-12646.

31. Onesti, S.; Miller, A. D.; Brick, P. Structure 1995, 3, 163-176.

32. Roy, H.; Zou, S. B.; Bullwinkle, T. J.; Wolfe, B. S.; Gilreath, M. S.; Forsyth, C. J.; Navarre, W. W.; Ibba, M. Nat Chem Biol 2011, 7, 667-669.

33. Park, M. H. J Biochem 2006, 139, 161-169.

34. Park, M. H.; Nishimura, K.; Zanelli, C. F.; Valentini, S. R. Amino Acids 2010, 38, 491-500.

35. Wolff, E. C.; Kang, K. R.; Kim, Y. S.; Park, M. H. Amino Acids 2007, 33, 341-350.

36. Murphey, R. J.; Gerner, E. W. J Biol Chem 1987, 262, 1503315036.

37. Glick, B. R.; Ganoza, M. C. Proc Natl Acad Sci U S A 1975, 72, 4257-4260.

38. Aoki, H.; Adams, S. L.; Turner, M. A.; Ganoza, M. C. Biochimie 1997, 79, 7-11.

39. Glick, B. R.; Ganoza, M. C. Eur J Biochem 1976, 71, 483-491.

40. Bullwinkle, T. J.; Zou, S. B.; Rajkovic, A.; Hersch, S. J.; Elgamal, S.; Robinson, N.; Smil, D.; Bolshan, Y.; Navarre, W. W.; Ibba, M. J Biol Chem 2013, 288, 4416-4423.

41. Ganoza, M. C.; Aoki, H. Biol Chem 2000, 381, 553-559.

42. Shimizu, Y.; Inoue, A.; Tomari, Y.; Suzuki, T.; Yokogawa, T.; Nishikawa, K.; Ueda, T. Nat Biotechnol 2001, 19, 751-755.

43. Kemper, W. M.; Berry, K. W.; Merrick, W. C. J Biol Chem 1976, 251, 5551-5557. 
44. Schreier, M. H.; Erni, B.; Staehelin, T. J Mol Biol 1977, 116, 727-753.

45. Saini, P.; Eyler, D. E.; Green, R.; Dever, T. E. Nature 2009, 459, 118-121.

46. Glick, B. R.; Chladek, S.; Ganoza, M. C. Eur J Biochem 1979, 97, 23-28.

47. Hersch, S. J.; Wang, M.; Zou, S. B.; Moon, K. M.; Foster, L. J.; Ibba, M.; Navarre, W. W. MBio 2013, 4.

48. Gutierrez, E.; Shin, B. S.; Woolstenhulme, C. J.; Kim, J. R.; Saini, P.; Buskirk, A. R.; Dever, T. E. Mol Cell 2013.

49. Zou, S. B.; Hersch, S. J.; Roy, H.; Wiggers, J. B.; Leung, A. S.; Buranyi, S.; Xie, J. L.; Dare, K.; Ibba, M.; Navarre, W. W. J Bacteriol 2012, 194, 413-425.

50. Park, J. H.; Johansson, H. E.; Aoki, H.; Huang, B. X.; Kim, H. Y.; Ganoza, M. C.; Park, M. H. J Biol Chem 2012, 287, 2579-2590.

51. Aoki, H.; Dekany, K.; Adams, S. L.; Ganoza, M. C. J Biol Chem 1997, 272, 32254-32259.

52. Abratt, V. R.; Mbewe, M.; Woods, D. R. Mol Gen Genet 1998, 258, 363-372.

53. Balibar, C. J.; Iwanowicz, D.; Dean, C. R. Curr Microbiol 2013. Apr 17. [Epub ahead of print].

54. Kaniga, K.; Compton, M. S.; Curtiss, R., III; Sundaram, P. Infect Immun 1998, 66, 5599-5606.

55. Bearson, S. M.; Bearson, B. L.; Brunelle, B. W.; Sharma, V. K.; Lee, I. S. Foodborne Pathog Dis 2011, 8, 725-732.

56. Charles, T. C.; Nester, E. W. J Bacteriol 1993, 175, 6614-6625.

57. Peng, W. T.; Banta, L. M.; Charles, T. C.; Nester, E. W. J Bacteriol 2001, 183, 36-45.

58. Ohashi, Y.; Inaoka, T.; Kasai, K.; Ito, Y.; Okamoto, S.; Satsu, H.; Tozawa, Y.; Kawamura, F.; Ochi, K. Biosci Biotechnol Biochem 2003, 67, 2245-2253.

59. Iannino, F.; Ugalde, J. E.; Inon de Iannino, N. Microb Pathog 2012, 52, 31-40.

60. Van Dyk, T. K.; Smulski, D. R.; Chang, Y. Y. J Bacteriol 1987, $169,4540-4546$.
61. Noinaj, N.; Guillier, M.; Barnard, T. J.; Buchanan, S. K. Annu Rev Microbiol 2010, 64, 43-60.

62. De Groote, M. A.; Granger, D.; Xu, Y.; Campbell, G.; Prince, R.; Fang, F. C. Proc Natl Acad Sci U S A 1995, 92, 6399-6403.

63. Sallee, N. A.; Rivera, G. M.; Dueber, J. E.; Vasilescu, D.; Mullins, R. D.; Mayer, B. J.; Lim, W. A. Nature 2008, 454, 1005-1008.

64. Holmes, A.; Muhlen, S.; Roe, A. J.; Dean, P. Infect Immun 2010, 78, 4445-4453.

65. Chen, L. M.; Kaniga, K.; Galan, J. E. Mol Microbiol 1996, 21, 1101-1115.

66. Hersh, D.; Monack, D. M.; Smith, M. R.; Ghori, N.; Falkow, S.; Zychlinsky, A. Proc Natl Acad Sci U S A 1999, 96, 2396-2401.

67. Fink, S. L.; Cookson, B. T. Cell Microbiol 2007, 9, 2562-2570.

68. Lara-Tejero, M.; Sutterwala, F. S.; Ogura, Y.; Grant, E. P.; Bertin, J.; Coyle, A. J.; Flavell, R. A.; Galan, J. E. J Exp Med 2006, 203, 1407-1412.

69. Miller, S. I.; Mekalanos, J. J. J Bacteriol 1990, 172, 2485-2490.

70. Bowden, S. D.; Rowley, G.; Hinton, J. C.; Thompson, A. Infect Immun 2009, 77, 3117-3126.

71. Zanelli, C. F.; Maragno, A. L.; Gregio, A. P.; Komili, S.; Pandolfi, J. R.; Mestriner, C. A.; Lustri, W. R.; Valentini, S. R. Biochem Biophys Res Commun 2006, 348, 1358-1366.

72. Zanelli, C. F.; Valentini, S. R. Amino Acids 2007, 33, 351-358.

73. Milon, P.; Rodnina, M. V. Crit Rev Biochem Mol Biol 2012, 47, 334-348.

74. Jackson, R. J.; Hellen, C. U.; Pestova, T. V. Nat Rev Mol Cell Biol 2010, 11, 113-127.

75. Jackson, R. J.; Hellen, C. U.; Pestova, T. V. Adv Protein Chem Struct Biol 2012, 86, 45-93.

76. Jordan, I. K.; Kondrashov, F. A.; Adzhubei, I. A.; Wolf, Y. I.; Koonin, E. V.; Kondrashov, A. S.; Sunyaev, S. Nature 2005, 433, 633-638.

Reviewing Editor: Alfred Wittinghofer 